\title{
On the use of electrostatic force microscopy as a quantitative subsurface characterization technique: A numerical study
}

\author{
C. Riedel, ${ }^{1,2,3, a)}$ A. Alegría, ${ }^{1,4}$ G. A. Schwartz, ${ }^{4}$ R. Arinero, ${ }^{5}$ J. Colmenero, ${ }^{1,2,4}$ \\ and J. J. Sáenz $z^{2,3}$ \\ ${ }^{1}$ Departamento de Física de Materiales UPVIEHU, Facultad de Química, Apartado 1072, 20080 \\ San Sebastián, Spain \\ ${ }^{2}$ Donostia International Physics Center, Paseo Manuel de Lardizábal 4, 20018 San Sebastián, Spain \\ ${ }^{3}$ Departamento de Física de la Materia Condensada and Instituto "Nicolás Cabrera," Universidad Autónoma \\ de Madrid, Campus de Cantoblanco, 28049 Madrid, Spain \\ ${ }^{4}$ Centro de Física de Materiales CSIC-UPVIEHU, Paseo Manuel de Lardizábal 5, 20018 San Sebastián, Spain \\ ${ }^{5}$ Institut d'Electronique du Sud (IES), UMR CNRS 5214, Université Montpellier II, CC 083, Place E. Bataillon, \\ 34095 Montpellier Cedex, France
}

(Received 17 May 2011; accepted 16 June 2011; published online 11 July 2011)

\begin{abstract}
We present a numerical study on the use of electrostatic force microscopy (EFM) as a non invasive subsurface characterization technique. We discuss the ability to resolve a buried object in a dielectric matrix considering two parameters: the detectability (i.e., signal superior to the noise) and the lateral resolution. The effects of the dielectric constant, thickness of the sample, and depth at which the object is buried are quantified. We show that the sensitivity reached in EFM permits to characterize subsurface objects in a dielectric matrix. We demonstrate that both lateral resolution and detectability decreases when the tip object distance increases. On the other hand, these two quantities increase with the dielectric constant of the matrix. A first step toward EFM tomography is proposed for objects creating non correlated signals. (C) 2011 American Institute of Physics. [doi:10.1063/1.3608161]
\end{abstract}

Electrostatic force microscopy (EFM) is a well known technique to characterize electrical and electrochemical properties of metals, semiconductors, dielectrics, and organic materials at the nanoscale. The lateral resolution of EFM has been largely studied at the surface of both metallic ${ }^{1-3}$ and dielectric samples ${ }^{4}$ and reaches nanometers values. In the DC mode and typical ambient condition, its great sensitivity allows measuring cantilever deflection of the order of the Ångström ${ }^{5}$ and frequency shift close to the hertz ${ }^{6}$ corresponding to hundreds of $\mathrm{pN}$ forces and tenths of $\mathrm{pN} / \mathrm{nm}$ force gradients, respectively. In their early work, Schönenberger et al. developed a polarizing optical interferometer with a sensitivity that permitted to monitor the recombination of single charge carriers. ${ }^{7}$ Today, the characterization of subsurface objects in dielectric matrix is of the upmost importance. Among the area focusing on this topic, we will cite the study of charge decay and lateral spreading in oxide ${ }^{8,9}$ that would allow further scale-down of nitride trap memory and the incorporation of nanostructured materials, such as carbon nanotubes (CNTs) into polymeric films to improve material performance. ${ }^{10-13}$ However, only few techniques permit to study subsurface properties: the optical microscope is limited by light wavelength and electron based techniques can damage the sample. EFM has recently been used to resolve CNTs in polymeric matrix. Jespersen et al. obtained a three dimensional mapping of individual CNT in a poly(methylmethacrylate) film ${ }^{14}$ whereas Zhao et al ${ }^{15}$ reported a complete experimental study of CNTs in polyimide nanocomposite films, anticipating EFM as a promising technique for this kind of characterization. Shen et al. ${ }^{16}$ used boundary

\footnotetext{
${ }^{\text {a) }}$ Author to whom correspondence should be addressed. Electronic mail: riedel@ies.univ-montp2.fr.
}

based methods to quantitatively investigate electrostatic signals, including a study of discrete charges on the sample surface or sheet charges inside a dielectric. They also developed a formalism to predict the formation of images in Kelvin probe force microscopy from a prescribed charge distribution and solve the charge distributions from the image (forward and reverse problems, respectively). ${ }^{17}$ In the following, we detail a numerical study that permits to understand the effect of the experimental parameters (dielectric constant and thickness of the sample, depth of the buried object) on the lateral resolution and detectability of subsurface objects using EFM. We will also demonstrate how the depth and the value of a point charge can be obtained.

The numerical simulation of the equivalent charge method $(E C M)^{6,18}$ or generalized image-charge $\operatorname{method}^{19}$ permits to compute the electric field, and, therefore, the additional force $\mathrm{F}$ and force gradient $\mathrm{G}$ (derivative of the force toward the tip sample distance $d_{0}$ ) created on the tip by a charge $\mathrm{q}_{0}$ in a dielectric. The idea of ECM is to find a discrete charge distribution ( $\mathrm{N}$ charge points $\mathrm{q}_{\mathrm{i}}$ at a distance $\mathrm{z}_{\mathrm{i}}$ on the axis $\mathrm{x}=0$ ) that represent the tip by creating a given potential $\mathrm{V}$ at the tip surface. $V_{0}^{i}$ and $V_{1}^{i}$ are the potentials created by the charge $\mathrm{q}_{\mathrm{i}}$ in the air $\left(\varepsilon_{0}\right)$ and in the dielectric $(\varepsilon)$, respectively. For each $\mathrm{q}_{\mathrm{i}}$, we introduce two series of images having a position and a value computed from the original position and value of the charge representing the tip (see references ${ }^{6,18}$ for more details). The core of the ECM is to find the value of the charges $\mathrm{q}_{\mathrm{i}}$ representing the tip that will satisfy the limit conditions $\left(V_{0}^{i}=V_{1}^{i}\right.$ and $\left.\varepsilon_{0} \frac{\partial V_{0}^{i}}{\partial z}=\varepsilon_{0} \varepsilon \frac{\partial V_{1}^{i}}{\partial z}\right)$ at the air/dielectric interface, and, $V_{1}^{i}=0$ at the dielectric/substrate interface). Once the electrostatic problem is solved, we introduce the charge $\mathrm{q}_{0}$ located inside the dielectric at a distance $\mathrm{r}_{0}$. In order to calculate the additional force on the tip, we will neglect the 
image charges induced on the tip due to the extra charge, i.e., we will assume that the charges in the tip are still given by the original set of $\mathrm{q}_{\mathrm{i}}$. For a semi infinite dielectric, the normal component of the electric field due to $\mathrm{q}_{0}$ in a point of coordinate $\mathrm{r}$ in the region above the surface is given by

$$
E_{z}^{q_{0}}=-\frac{1}{4 \pi \varepsilon}\left(\frac{2 \varepsilon_{0}}{\varepsilon+\varepsilon_{0}} q_{0}\right) \frac{\partial}{\partial z}\left(\frac{1}{\left|r-r_{0}\right|}\right) .
$$

For the case of real finite sample, this approach remains valid as long as the contribution of the substrate is negligible. We have checked that this contribution was inferior to $5 \%$ in all the data presented in this paper. Fig. 1(a) exemplifies this calculation by representing the additional force created by a single charge $\mathrm{q}_{0}=1.6 \times 10^{-19} \mathrm{C}$ in a medium having a dielectric constant $\varepsilon=4$ and a thickness $\mathrm{h}=500 \mathrm{~nm}$ under a difference of voltage of $1 \mathrm{~V}$ between the tip and the counter electrode. The tip is simulated with classical experimental parameters: radius of $\mathrm{R}=30 \mathrm{~nm}$, half cone angle $\theta=15^{\circ}$, and tip sample distance $\mathrm{d}_{0}=10 \mathrm{~nm}$. Both samples and geometric parameters will hold for the entire study, unless otherwise specified. The value of the force is recorded at different depths $-\mathrm{Z}=[2,10,50,100$, and 150] $\mathrm{nm}$ (dashed lines) and plotted in Fig. 1(b). Fig. 1(c) represents the profiles of the force recorded just below the surface at $\mathrm{z}=-2 \mathrm{~nm}$ for different dielectric constants. Similar plots are obtained for the force gradient. Fumagali et al. have studied the volume of an insulator probed through the evaluation of the difference in the force density on the substrate with the probe at near and far distance from the surface. ${ }^{20}$ In this manuscript, we will focus on two important parameters that appear in the profiles recorded in Figs. 1(b) and 1(c): the half width at half maximum that is defined as the lateral resolution and the maximum value of the signal (in $x=0$ ), that will determine the level of detectability.

We will first discuss the variation of the lateral resolution as a function of the experimental parameters and the possibility to determine the depth and value of a point charge. The maximum intensity decreases when both absolute depth (Fig. 1(b)) and dielectric constant (Fig. 1(c)) increase. However, we observe a broadening and a narrow-
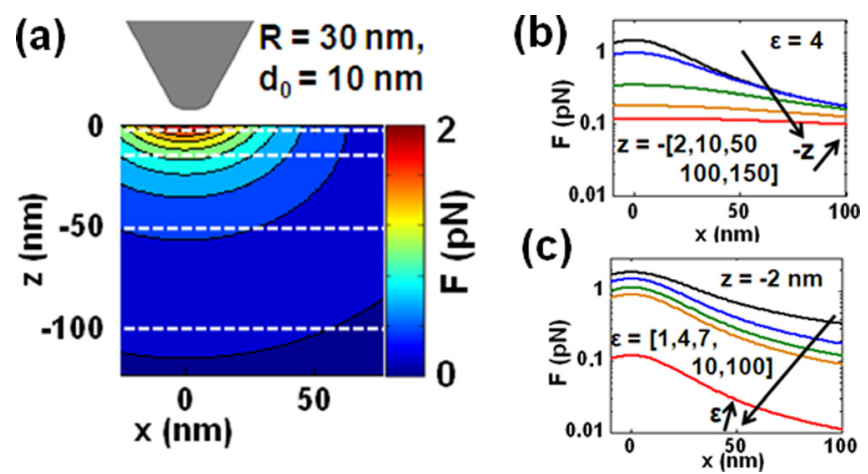

FIG. 1. (Color online) (a) Value of the force created by a single charge in a medium having a dielectric constant of 4 and a thickness of $500 \mathrm{~nm}$ under a difference of voltage of $1 \mathrm{~V}$ between the EFM tip and the counter electrode. (b) Force recorded along the dashed lines in (a), situated at a depth $\mathrm{z}=-[2$, $10,50,100$, and 150] $\mathrm{nm}$ for $\varepsilon=4$. (c) Force recorded along the line situated at a depth $\mathrm{z}=-2 \mathrm{~nm}$ for different values of the dielectric constant $\varepsilon=[1,4$, 7,10 , and 100]. ing of the curves in Figs. 1(b) and 1(c), respectively. These two different behaviors are represented in Fig. 2 where the lateral resolution is represented as a function of the depth for $\varepsilon=4$ (Fig. 2(a)) and as a function of the dielectric constant at $\mathrm{z}=-2 \mathrm{~nm}$ (Fig. 2(b)).

In Fig. 2(a), we observe that the value of the lateral resolution increases linearly with the depth for both the force and gradient. Therefore, even if the detection of an object buried far from the surface is possible, EFM will not permit a high resolution imaging. In Fig. 2(b), the value of the lateral resolution decreases to the limit value of a metallic surface $(\varepsilon \rightarrow \infty)$ when the dielectric constant increases. This result is in agreement with previous simulation at the surface of the sample. ${ }^{4}$ If the setup is sensitive enough to detect object inside a high dielectric constant matrix, they will be resolved with a good lateral resolution. As it will be shown later for the detectability, when the sample is thick enough $(\mathrm{h}>500$ $\mathrm{nm})$, the total dielectric thickness has almost no effect on the lateral resolution. As for the case of object at the surface of the sample ${ }^{1-4}$ due to shorter interaction range, the resolution is better in the gradient mode than in the force mode.

Let us now consider the situation pictured in Fig. 2(c). We scan the sample over two charges having a value of $\mathrm{q}_{0}$ and $5 q_{0}$, buried at 2 and $50 \mathrm{~nm}$ below the surface of the insulator presented in Fig. 1(a), respectively. We assume that the charges are separated enough to create non correlated signals. Fig. 2(d) presents the profile of the force recorded in this situation. From the value of the half width at half maximum, we are able to compute the depth at which the charges are burried, $-z_{0}$, from Fig. 2(a). Once $-z_{0}$ is known, the value of the charge can be calculated from the ratio between the maximum of the curves in Fig. 2(d) and the value of $\mathrm{F}\left(-\mathrm{z}_{0}\right)$ simulated for one charge presented in the Fig. 3(a), which will be introduced later. When signals of different charges overlap, realizing tomography with EFM is more complicated. One possible approach would consist in a deconvolution of the signal recorded at different tip sample distances as it is currently being developed for volume-scanning near-field microscopy. ${ }^{21}$
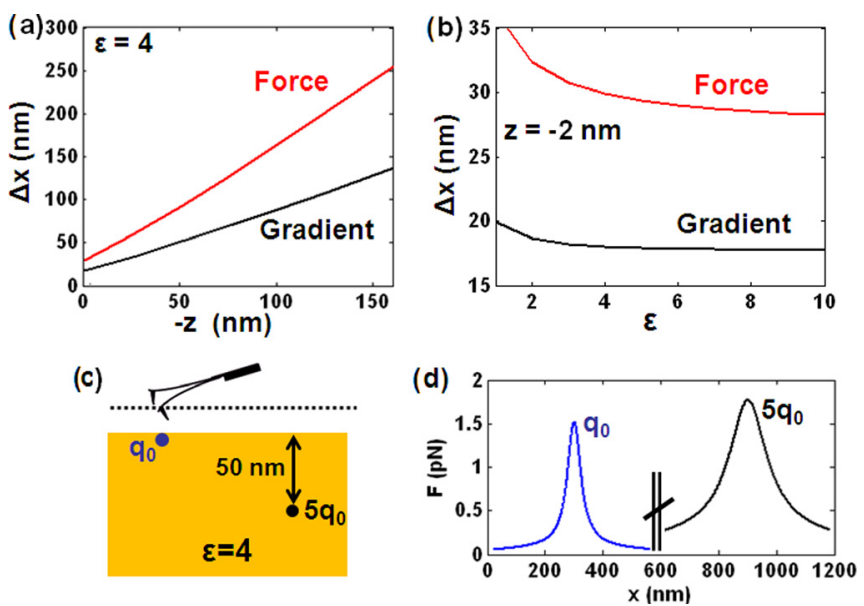

FIG. 2. (Color online) $\mathrm{R}=30 \mathrm{~nm}$ and $\mathrm{d}_{0}=10 \mathrm{~nm}$. Lateral resolution in the force and gradient mode as a function of: (a) the depth and (b) the dielectric constant. (c) Schematic representation of a scan over two point charges buried at 2 and $50 \mathrm{~nm}$ in a sample having a dielectric constant $\varepsilon=4$. Values of the charges are $q_{0}$ and $5 q_{0}$, respectively. (d) Profile of the force recorded in (c). 
(a) (b)
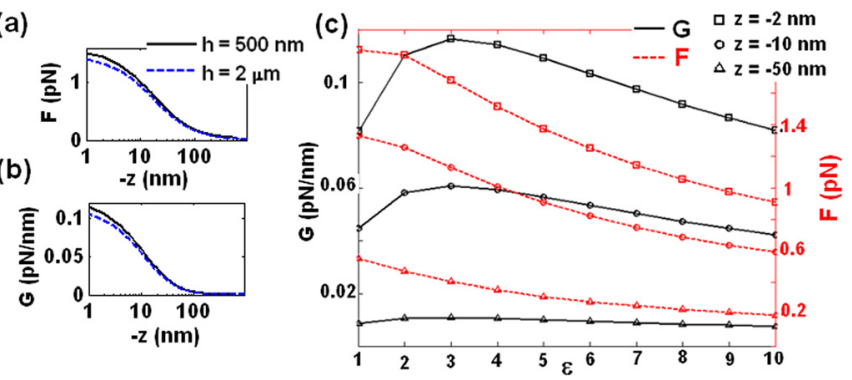

FIG. 3. (Color online) (a) Force and (b) gradient recorded below the tip inside the sample (along the $\mathrm{x}=0$ axis in Fig. 1(a)). (c) Force and gradient recorded at different depth (at $\mathrm{x}=0$ ) as a function of the dielectric constant.

We will now focus our study on the capability of EFM to detect charges, considering the value of the force and force gradient below the tip (over the $\mathrm{x}=0$ axis in Fig. 1). The quantitative values of force and force gradient here presented, given for one buried charge under a difference of voltage of $1 \mathrm{~V}$ between the tip and the counter electrode, are to be compared to the experimental noise depending on each particular instrument, experimental conditions, and mode. The deflection of the cantilever and frequency shift $\Delta \mathrm{f}$ are directly related to the force $\mathrm{F}=-\mathrm{kd}$, and force gradient $\mathrm{G} \approx-2 \mathrm{k} \Delta \mathrm{f} / \mathrm{f}_{0}$ where $\mathrm{k}$ and $\mathrm{f}_{0}$ are the stiffness and resonance frequency of the cantilever. In the DC gradient mode and ambient condition, typical noises in cantilever deflection and frequency shift are of the order of the Ångström ${ }^{5}$ and hertz ${ }^{6}$ corresponding to hundreds of $\mathrm{pN}$ forces and tenths of $\mathrm{pN} / \mathrm{nm}$ force gradients, respectively. An object will be detectable if the additional force or force gradient created on the tip are superior to the noise levels. An easy experimental way to increase the signal in EFM is to increase the voltage between the tip and the counter electrode.

Figs. 3(a) and 3(b) represent the force and force gradient recorded as a function of the depth, respectively, below the tip (over the $\mathrm{x}=0$ axis in Fig. 1) for two samples having a thickness of $500 \mathrm{~nm}$ and $2 \mu \mathrm{m}$. We observe that both force and force gradient decrease with the same trend for samples having total thicknesses of $500 \mathrm{~nm}$ and $2 \mu \mathrm{m}$. When the sample is thick enough, the total thickness does not affect the detectability. The second point is that the ratio between the signal created by a charge and the typical noise level is superior in the gradient mode that, therefore, presents a better sensitivity. For example, $2 \mathrm{~nm}$ below the surface, this ratio has a value of 1/100 in the force mode and 1 in the gradient mode, respectively. We remind that these numbers are given for the signal created by a single charge having a value $\mathrm{q}_{0}=1.6 \times 10^{-19} \mathrm{C}$ under a difference of voltage of $1 \mathrm{~V}$. In typical experimental conditions, for $\mathrm{V}=10 \mathrm{~V}$, the $\mathrm{DC}$ force gradient mode allows detection of single carrier buried down to hundreds of nanometers whereas DC force only permits detection of objects having stronger charge (i.e., an object situated at $10 \mathrm{~nm}$ below the surface, having a charge of $10 \mathrm{q}_{0}$ under a voltage of $10 \mathrm{~V}$ ).

The behaviour of both maximum of the force and force gradient $($ at $\mathrm{x}=0)$ as a function of the dielectric constant is presented in Fig. 3(c) for different depths. For the force, these numbers correspond to the ones of the maximum of
$\mathrm{F}(\mathrm{x})$ presented in Fig. 1(c). As expected, when the dielectric constant increases, the value of the force created by a charge at a constant depth inside the dielectric decreases. It is worth noting in Fig. 1(c) that the value of the force created by a charge just below the surface of a sample having a dielectric constant of 100 is still of the order of $0.1 \mathrm{pN}(0.01 \mathrm{pN} / \mathrm{nm}$ for the force gradient, data here not presented) and would, therefore, allow a detection with a sensitive system. This screening effect is expected for the force, however, in Fig. 3 (c), we note a non monotonous variation in the force gradient that presents a maximum: in this mode, the stronger signal is obtained for an intermediate value of the dielectric constant. In agreement with Figs. 3(a) and 3(b), we note that the intensity of both force and force gradient curves decreases when the tip-object distance increases.

In summary, we have presented a study of the detectability and lateral resolution of buried objects in dielectrics using EFM. The numerical value of the lateral resolution decreases when the dielectric constant of the matrix increases. Lateral resolution and sensitivity (i.e., ratio signal over noise) are better in the DC gradient than force mode. Even if the detection of objects buried far from the surface is possible, due to a monotonic dependence of the lateral resolution on the tip-object distance, high resolution imaging will only be possible close to the surface. Finally, the intensity of the signal decreases monotonously with the dielectric constant in the force mode whereas a maximum appears in the force gradient. The quantitative analysis here presented show that EFM is a reliable technique to characterize subsurface objects in a dielectric matrix. We proposed a first step toward tomography for samples presenting "dilute" point charges creating non correlated signals by the interpretation of both maximum and half width at half maximum of the signal recorded.

We acknowledge the Basque Country Government (project IT-436-07), the Comunidad de Madrid Microseres-CM Project (S2009/TIC-1476), the Spanish Ministry of Science and Innovation (Grant Nos. MAT 2007-63681 and FIS200913430-C01-C02), and the ESMI Program.

\footnotetext{
${ }^{1}$ S. Belaidi et al., Appl. Phys. A: Mater. Sci. Process. 66, S239 (1998).

${ }^{2}$ Y. Shen et al., Nanotechnology 19, 035710 (2008).

${ }^{3}$ S. Gomez-Monivas et al., Nanotechnology 12, 496 (2001).

${ }^{4}$ C. Riedel et al., Nanotechnology 22, 285705 (2011).

${ }^{5}$ G. Gramse et al., Nanotechnology 20, 395702 (2009).

${ }^{6}$ C. Riedel et al., J. Appl. Phys. 106, 024315 (2009).

${ }^{7}$ C. Schonenberger and S. F. Alvarado, Phys. Rev. Lett. 65, 3162 (1990).

${ }^{8}$ F. Marchi et al., J. Electrostat. 66, 538 (2008).

${ }^{9}$ S. D. Tzeng and S. Gwo, J. Appl. Phys. 100, 023711 (2006).

${ }^{10}$ T.-W. Chou, L. Gao, E. T. Thostenson, Z. Zhang, and J.-H. Byun, Compos. Sci. Technol. 70, 1 (2010).

${ }^{11}$ H. Cebeci et al., Compos. Sci. Technol. 69, 2649 (2009).

${ }^{12}$ O. Breuer and U. Sundararaj, Polym. Compos. 25, 630 (2004).

${ }^{13}$ W. Bauhofer and J. Z. Kovacs, Compos. Sci. Technol. 69, 1486 (2009).

${ }^{14}$ T. S. Jespersen and J. Nygard, Appl. Phys. Lett. 90, 183108 (2007).

${ }^{15}$ M. Zhao et al., Nanotechnology 21, 225702 (2010).

${ }^{16}$ Y. Shen et al., Eng. Anal. Boundary Elem. 32, 682 (2008).

${ }^{17}$ Y. Shen et al., Rev. Sci. Instrum. 79, 023711 (2008).

${ }^{18}$ C. Riedel et al., Eur. Phys. J. Appl. Phys. 50, 10501 (2010).

${ }^{19}$ G. M. Sacha, E. Sahagun, and J. J. Saenz, J. Appl. Phys. 101, 024310 (2007).

${ }^{20}$ L. Fumagalli et al., Appl. Phys. Lett. 96, 183107 (2010).

${ }^{21}$ J. Sun et al., Appl. Phys. Lett. 95, 121108 (2009).
} 